

\title{
La educación estética de profesionales de la educación en formación inicial desde la extensión universitaria
}

\author{
Luis Enrique Díaz Peralta ${ }^{1}$ \\ Universidad Central "Marta Abreu” de Las Villas \\ Cuba \\ 1dias@uclv.cu \\ Eduardo Alejandro Hernández Alfonso ${ }^{2}$ \\ Universidad Central "Marta Abreu” de Las Villas \\ Cuba \\ ealejandro@uclv.cu \\ Luis Ernesto Paz Enrique ${ }^{3}$ \\ Universidad Central "Marta Abreu" de Las Villas \\ Cuba \\ luisernestope@uclv.cu
}

\begin{abstract}
Resumen
La formación integral de profesionales de la educación resulta esencial para cumplir su encargo social. En este proceso formativo, la educación estética ha de ser un contenido esencial por su alcance universal. Desde la extensión universitaria, se puede facilitar la educación estética para contribuir a la apreciación y comprensión de la sociedad. Con base en las encuestas de satisfacción estudiantil efectuadas en la Universidad Central "Marta
\end{abstract}

\section{(9)( $\Theta \Theta$}

Recibido: 5 de octubre de 2018. Aprobado: 10 de abril de 2019.

http://dx.doi.org/10.15359/rep.14-1.10

1 Doctor en Ciencias Pedagógicas

2 Licenciado en Comunicación Social. Doctorante en Ciencias Sociológicas

3 Licenciado en Ciencias de la Información. Doctorante en Ciencias Sociológicas 
Abreu" de Las Villas durante el curso escolar 2017-2018, se constatan insuficiencias en el desarrollo de profesionales de la educación en formación inicial. Los resultados anteriores arrojan como principales causas, la falta de conocimientos teóricos por parte de quienes se encargan del proceso enseñanza-aprendizaje acerca de la extensión universitaria, y la carencia de un diseño integrador de actividades que favorezca los contenidos de la educación estética. Se propone como objetivo: identificar los rasgos que caracterizan la educación estética de profesionales de la educación en formación inicial desde la extensión universitaria en la Universidad Central "Marta Abreu" de Las Villas, Cuba. La inclusión de contenidos sobre la educación estética para profesionales de la educación en formación permite el acercamiento a los valores artísticos y estéticos, y favorece la inserción en la actividad estética y en la crítica del arte como vía para una interpretación exhaustiva de la sociedad.

Palabras clave: Formación integral, educación, extensión universitaria, estética.

\begin{abstract}
The integral training of students is essential in higher education. In this formative process, aesthetic education must be an essential content because of its universal scope. From university extension, aesthetic education can be facilitated by contributing to the appreciation and understanding of society. Based on the student satisfaction surveys conducted at the Universidad Central "Marta Abreu" de Las Villas, Cuba during the 2017-2018 school year, there are insufficiencies in the development of pre-service education professionals' training. The previous results show, as main causes, the lack of theoretical knowledge on the part of those in charge of the teaching-learning process about university extension and the lack of an integrating design of activities that favors the contents of aesthetic education. The objective of this paper is to identify the features that characterize aesthetic education of pre-service education professionals' training from the university extension at the Universidad Central
\end{abstract}


"Marta Abreu" de Las Villas, Cuba. The inclusion of content on aesthetic education for pre-service education professionals allows the approach to artistic and aesthetic values. They favor the insertion in the aesthetic activity and in the criticism of art as a way for an exhaustive interpretation of society.

Keywords: comprehensive education, education, university extension, aesthetics

\section{Introducción}

En el mundo contemporáneo, se manifiestan enormes contrastes como resultado de los efectos de la globalización neoliberal: por una parte, el extraordinario desarrollo de la ciencia y el conocimiento; por otra, las tendencias de la evolución económica-social. Esta lucha antagónica entre el establecimiento de la cultura única occidental y la preservación de las identidades nacionales de los países subdesarrollados, constituidas por un proceso esencialmente cultural, que ha ido definiendo cualitativamente las capacidades y posibilidades de análisis y percepción de la ciudadanía sobre los más complejos fenómenos de la economía, la naturaleza y las relaciones sociales.

Existe un consenso universal sobre la universidad, pues se entiende desde su función educativa y la vez formativa. Garza y Galo (2010) plantean que la universidad "para desempeñar un buen papel en este mundo cambiante debe revalorar su participación en la cohesión social" (p. 77). Las universidades deben fomentar los procesos sustantivos desde una visión holística que permita integrar la docencia, la investigación y la extensión universitaria.

La extensión universitaria constituye uno de los procesos sustantivos de la universidad que contribuye a la formación del profesional; posibilita una verdadera interrelación con la comunidad intra y extrauniversitaria y facilita la promoción de la cultura a partir de un bagaje intelectual alcanzado con el desarrollo armónico de los contenidos de la formación integral. En ello, la educación estética ha de ser un contenido esencial por su alcance universal, cosmovisión y por los nexos dialécticos que establece con el resto de los contenidos de la formación integral: Desarrolla la apreciación, la aprehensión de lo bello, de lo sublime y su repercusión en el desarrollo de conductas estéticas en el 
respeto a la ciudadanía, a la familia, a la profesión, a la nación y al amor por la naturaleza.

La educación estética del estudiantado egresado universitario es particularmente importante para quienes tendrán el encargo social de educar en la sociedad. El análisis en torno a esta temática debe ser preocupación de personal directivo, docente e investigador que participa en la formación de profesionales de la educación.

Suárez (1982), Rodríguez, Balmaceda y Abello (2015) y Ubals (2012) profundizan en la relación educación artística- educación estética y critican la reducción de su objeto de estudio. El interés principal ha constituido el perfeccionamiento de la labor extensionista en las universidades, que abordan la educación estética como un contenido a desarrollar. En sentido general, se dirigen hacia los siguientes aspectos:

- Caracterizan la extensión universitaria como un proceso universitario y sus relaciones esenciales como sistema, lo que permite demostrar que constituye una función sustantiva de la universidad y un proceso universitario que tiene como objetivo promover la cultura en sentido general y en particular la educación estética.

- Fundamentan la extensión universitaria como un factor vinculante entre la universidad y la sociedad.

- Abordan las categorías de educación estética y educación artística desde diferentes perspectivas críticas, precisando sus relaciones y diferencias.

- Consideran la educación estética como objetivo de la extensión universitaria y componente de la formación profesional en la educación superior.

- Brindan propuestas de acciones para el trabajo cultural en general sobre bases científicas y pedagógicas.

- Conciben importantes referentes teóricos y metodológicos que permiten conocer la evolución y desarrollo del proceso extensionista de manera general y en particular de la educación estética en el mundo, América Latina y en Cuba.

De forma general, se constatan, en las encuestas de satisfacción estudiantil, carencias en personal directivo y docente encargados de dirigir el proceso formativo. Estas se manifestaron en la subvaloración 
del papel que desempeña la educación estética en la labor educativa y la formación integral, la poca sistematización de los contenidos teóricos referentes a la educación estética de profesionales en formación inicial desde la extensión universitaria, la falta de una proyección integral para alcanzar este objetivo, así como la insuficiente intencionalidad del trabajo con la educación estética en las estrategias educativas de las carreras, los años y proyectos educativos de los grupos. Port tanto, se plantea, como objetivo de la investigación, identificar los rasgos que caracterizan la educación estética de profesionales de la educación en formación inicial desde la extensión universitaria en la Universidad Central "Marta Abreu" de Las Villas, Cuba.

\section{Metodología}

La investigación clasifica como descriptiva con aporte teórico. Se centra en identificar los presupuestos teóricos para fundamentar los rasgos que caracterizan la educación estética de profesionales de la educación en formación inicial desde la extensión universitaria en la Universidad Central "Marta Abreu" de Las Villas, Cuba. Se emplean métodos en el nivel teórico y empírico para la recogida de los datos. Del nivel teórico se utilizan el histórico-lógico, analítico-sintético, inductivo-deductivo, el sistémico-estructural:

El histórico-lógico permitió obtener los referentes imprescindibles sobre el devenir histórico de la educación estética, la extensión universitaria y la formación de profesionales en Cuba de manera general y de profesionales de la educación en particular, así como revelar las potencialidades de la extensión universitaria para contribuir a la educación estética.

El analítico-sintético posibilita una constante composición y recomposición de las ideas para la sistematización de la información sobre los procesos de formación de profesionales de la educación, de extensión universitaria y sobre la educación estética como contenido de la formación general integral.

El inductivo-deductivo permitió determinar regularidades y realizar generalizaciones, referidas fundamentalmente con las potencialidades de las actividades de extensión universitaria para contribuir a la educación estética de profesionales de la educación en formación inicial, la determinación de los rasgos fundamentales que se derivan de 
la operacionalización de las definiciones de extensión universitaria y educación estética.

El sistémico-estructural permitió develar la relación de la educación estética con los demás procesos universitarios que intervienen en la formación de profesionales de la educación en formación inicial y de los cambios que se van produciendo en la educación estética del futuro estudiantado egresado.

En el nivel empírico se empleó el análisis documental clásico. La técnica empleada que facilita la recogida de información es la revisión de documentos, permite la localización de referentes teóricos acerca de la temática a partir del análisis documental. En el análisis documental se emplearon diversos materiales que datan de la década del 40 hasta la actualidad, sin perder de vista el análisis del contexto histórico, lógico y social. Se utiliza la información que brinda cada documento. Para garantizar la veracidad, credibilidad y cientificidad de la investigación a partir de la recogida de información, se recurre a la triangulación de datos.

\section{Resultados y discusión}

\section{Características del proceso de formación de profesionales de la educación en formación inicial}

La universidad contemporánea, fruto y reflejo de los cambios económicos, políticos y sociales, se diferencia, en principio, de la universidad que le antecedió. El carácter casi privativo de acumular los conocimientos y preparar a los individuos para ejercer profesionalmente durante su vida se ha modificado esencialmente. Esta realidad impone a las universidades el reto de atender la formación integral de sus estudiantes, que en síntesis implica:

la necesidad de lograr un profesional creativo, independiente, preparado para asumir su autoeducación durante toda la vida; que sea capaz de mantenerse constantemente actualizado, utilizando igualmente las oportunidades ofrecidas por las universidades de atender al profesional con una educación posgraduada que responda a las necesidades del desarrollo del país. (...) La educación superior cubana ha asumido esa idea de formación integral, elevándola al rango de idea rectora principal. (Horruitiner, 2007, p. 3) 
A tenor con lo anterior, el ideal de formación integral del individuo está indisolublemente ligado a la formación de la nacionalidad y al desarrollo de las concepciones pedagógicas más avanzadas de los siglos XIX y XX. Los centros formadores de profesionales de la educación exigen para su cumplimiento que todos los entes implicados sean portadores de la teoría y la práctica pedagógica. Es necesario, además, el conocimiento de las demandas de la sociedad para poder desempeñar una labor formativa coherente. Es necesario tener en cuenta cómo la formación, el desarrollo, la instrucción, la educación y los procesos de enseñanza aprendizaje juegan un rol fundamental en la educación estética de las generaciones futuras de profesionales de la educación.

El término formación es una categoría pedagógica que ha sido objeto de varias interpretaciones por las investigaciones. Este aparece para referirse al proceso y resultado del conjunto de hechos que conciernen a la formación y expresa la función evolutiva del ser humano, en el proceso de asimilación de conceptos, hábitos y habilidades, y al proceso sustantivo universitario, que unido al de investigación y extensión universitaria, garantizan la formación integral de las generaciones futuras de profesionales.

Chávez, Suárez y Permuy (2003) abordan que "se entiende por formación al nivel que alcance un sujeto en cuanto a la explicación y comprensión que tenga de sí mismo y del mundo material y social" ( $p$. 46). Álvarez (1990) refiere que formación "es el proceso y el resultado cuya función es la de preparar al hombre en todos los aspectos de su personalidad" (p. 169). Savin (1978) la define como el "proceso de formación de la personalidad y su perfeccionamiento en el transcurso de la vida y también como resultado del desarrollo y la educación" (p. 17).

Se coincide con Valle Lima (2012) que define de manera específica la formación en el contexto de profesionales de la educación el siguiente proceso:

Concibe, desarrolla, y evalúa sistemas de actividades y de relaciones para lograr en la formación del profesional de la educación una correcta dirección del proceso pedagógico y generar un auto perfeccionamiento de su actividad pedagógica, en correspondencia con las exigencias de la sociedad. (Valle Lima, 2012, p. 202) 
En consonancia con lo anterior, el destacado pedagogo Boldiriev (1982) explica que "desarrollar integralmente al individuo significa desarrollar sus fuerzas físicas y espirituales" (p. 5). En la pedagogía, su análisis se manifiesta en estrecha relación con la categoría desarrollo, pues, a decir de Chávez, Suárez y Permuy (2003), "la formación del sujeto como personalidad no se da aislada del desarrollo y este conduce, en última instancia, a un nivel psíquico superior" (p. 46). Savin (1978) la considera como el proceso de desarrollo espiritual, del perfeccionamiento y el cambio cualitativo en todas las esferas importantes de la personalidad, en el reflejo de la realidad que la rodea, en la actitud ante los fenómenos y ante los seres humanos, así como en los procesos cognitivos.

La sociedad actual impone a las universidades la responsabilidad de egresar profesionales integrales capaces de resolver los complejos problemas que deben afrontar. Deben caracterizarse, fundamentalmente, por el enfoque integral de campos del conocimiento, armonizar las dimensiones de los procesos sustantivos de la universidad (la formación, la investigación y la extensión universitaria) y por el indisoluble nexo existente entre las dimensiones del proceso de formación, expresado en sus dos ideas rectoras: la unidad entre la educación y la instrucción, y la vinculación del estudio con el trabajo.

La formación integral de profesionales de la educación requiere del desarrollo armónico de todos los procesos universitarios, así como de los contenidos que integran cada uno de ellos. La educación estética como uno de los contenidos que intervienen en la formación integral debe desarrollar la sensibilidad estética de profesionales de la educación en formación inicial, para que, una vez que se gradúen, puedan extender su influencia a la escuela, la familia y la comunidad. Ello facilitará cumplir con la educación estética de sus miembros sobre la base de la armonía, el comportamiento, el amor al arte y a la naturaleza.

La formación integral de profesionales de la educación demanda el indisoluble nexo existente entre las dimensiones instructiva, educativa y desarrolladora del proceso de formación expresado en sus dos ideas rectoras: la unidad entre la educación y la instrucción, y la vinculación del estudio con el trabajo. La unidad de la instrucción y la educación constituye una idea rectora del proceso de formación de estos grupos profesionales y un problema fundamental de la pedagogía en nuestros días. Su contenido esencial es el desarrollo de una personalidad capaz de favorecer el progreso social e individual. 
Se manifiesta una relación dialéctica entre la instrucción y la educación. Al respecto, Valdés (1981) expresó que no se puede "considerar la instrucción en divorcio con la educación; aquella con esta es el proceso por el cual las fuerzas individuales se desarrollan y se armonizan; pero a su vez la educación, recorriendo caminos semejantes alcanza un término idéntico" (p. 105). La enseñanza es una categoría estrechamente relacionada con la instrucción y la educación. Los autores Salcedo (2012), Vidal y Fernández (2015) coinciden en que la enseñanza-aprendizaje debe entenderse como un proceso bilateral.

La educación constituye un proceso que incluye la aprehensión de conocimientos, pero también la incorporación de habilidades para desarrollarlos. En el caso particular de la extensión universitaria, se trata de identificar sus potencialidades educativas de este proceso sustantivo de la universidad e incorporarlas al proceso de formación. Esto contribuye a la formación de valores en general y de la educación estética en particular, para un desempeño exitoso del profesional de la educación.

La formación de profesionales de la educación es un ejemplo fehaciente de un transformador enfoque de lo laboral con un modelo de formación desde el trabajo. Se puede afirmar que la formación integral de profesionales de la educación requiere del desarrollo armónico de todos los procesos universitarios, así como de los contenidos que integran cada uno de ellos. La extensión universitaria constituye un proceso esencial en la universidad para la educación estética de profesionales de la educación en formación inicial, como uno de los contenidos que intervienen en ello. Este proceso sustantivo posee las condiciones para la implementación de actividades que despierten la sensibilidad, a la vez que les dota de las herramientas necesarias para su labor de transformación estética de la escuela y la comunidad.

Para alcanzar este objetivo, la universidad debe lograr que la extensión universitaria adquiera el mismo nivel de desarrollo que los procesos de formación e investigación. Su enfoque integral debe concretarse en un proyecto educativo que identifique y aborde la solución de las principales necesidades educativas. A partir de un diagnóstico grupal e individual que posibilite atender las necesidades estéticas de sus integrantes para garantizar los resultados desde fundamentos científicos.

Los elementos fundamentales que caracterizan la educación estética y que se asumen en la investigación se basan en: 
- La educación estética es el proceso educativo conscientemente organizado, dirigido y sistemático, que se ejerce sobre la personalidad.

- La educación estética se desarrolla mediante la actividad y la comunicación, que se establece en la transmisión de los conocimientos y experiencias acumuladas por la humanidad.

- La educación estética está encaminada a la formación y desarrollo de una actitud estética, la capacidad y habilidades para la percepción, valoración y creación estética en todas las esferas de la vida material y espiritual de la sociedad socialista.

- La educación estética es un contenido de la formación integral de la personalidad.

Consecuentemente, el profesorado y los maestros y maestras deben poseer educación estética, pues tienen la alta responsabilidad de contribuir a la educación de las demás personas. Esta necesidad fue expresada por Estévez (2008) al manifestar que "las cualidades para apreciar, los gustos estéticos, crear lo bello en el arte y la realidad, son rasgos indispensables del hombre universalmente desarrollado; pero es necesario educarlas, ya que no se manifiestan por sí solas" (p. 62).

En el caso de las universidades, es necesario desarrollar esas cualidades a lo largo de toda la formación y a través de todos los procesos. La formación, la investigación y la extensión universitaria en su interrelación permitirán egresar profesionales de alta calidad, con elevados valores profesionales y morales, con amplia cultura y capaces de transformar la sociedad. La extensión universitaria se asume como un proceso universitario, de interacción humana. Una función de la universidad y un proceso universitario formativo, una función totalizadora, integradora y dinamizadora.

A partir de lo anterior, se prestó mayor atención al desarrollo de la capacidad de comprensión de lo bello en la realidad, en los objetos y en los medios expresivos del arte, a través de las actividades de extensión universitaria. La extensión universitaria y la educación estética son procesos interrelacionados. La extensión universitaria constituye una vía fundamental para la educación estética. Desde sus contenidos específicos ofrece las formas organizativas (verbales, prácticas y visuales) para el desarrollo de la educación estética como contenido de la educación integral. 
La extensión universitaria posee las condiciones para la implementación de actividades que despierten la sensibilidad estética, a la vez que concede a los grupos de profesionales de la educación en formación inicial las herramientas necesarias para su labor de transformación estética de sus estudiantes, la escuela y la comunidad. A su vez, la educación estética contribuye a la formación de capacidades de apreciación, de gustos y de la sensibilidad estética que se necesita para el desarrollo eficaz de los contenidos de la extensión universitaria.

\section{Conclusiones}

Los referentes teórico-metodológicos de la educación estética de profesionales de la educación en formación inicial desde la extensión universitaria parten de la concepción humanista de los procesos de enseñanza aprendizaje. Estos tienen como aspiración la formación integral de los individuos, donde la educación estética constituye uno de sus contenidos fundamentales, que puede ser potenciada desde la extensión universitaria por su carácter integrador.

Se constatan carencias en la educación estética manifestadas en la subvaloración del papel que desempeña en la labor educativa y la formación integral, la poca sistematización de los contenidos teóricos sobre esta, así como la insuficiente intencionalidad del trabajo con ella en las estrategias educativas. Se identifican, entre las principales causas centradas en la falta de conocimientos teóricos acerca de la educación estética, sus rasgos distintivos, su contenido y cómo desarrollarla, la carencia de un diseño integrador de actividades y una visión esquemática del proceso de extensión universitaria.

Las transformaciones que se producen en la educación estética de profesionales de la educación en formación mediante la implementación de actividades desde la extensión universitaria son el acercamiento a los valores artísticos y estéticos. Estos potencian un incremento de la inserción en la actividad estética y en la crítica del arte; se constituyen en dinamizadores del desempeño profesional y favorecedores de la integración creadora de los conocimientos adquiridos, así como de la participación activa en la socialización y divulgación de los contenidos estéticos. 


\section{Referencias}

Álvarez, C. (1990). Fundamentos teóricos de la dirección del proceso docente en la educación superior. La Habana: Empresa Nacional de Producción.

Boldiriev, N. (1982). Metodología de la organización del trabajo educativo. La Habana: Pueblo y Educación.

Chávez, J., Suárez, A. y Permuy, L. (2003). Acercamiento a la pedagogía general. La Habana: ICCP.

Estévez, P. (2008). Los colores del arco iris. La Habana: Editorial Pueblo y Educación.

Garza, R. y Galo, J. (2010). La sustentabilidad en las instituciones de educación superior: Una visión holística. Monterrey: Ediciones La\&Go.

Horruitiner, P. (2007). La universidad cubana: El modelo de formación. Revista Pedagogía Universitaria, 12(4), 15-31.

Rodríguez, 1., Balmaseda, O. y Abello, A. (2015). Nueva ortografía para todos. La Habana: Pueblo y Educación.

Salcedo, F. (2012). Papel del profesor en la enseñanza de estrategias de aprendizaje.Didasc@lia: Didáctica y Educación, 3(3),17-28.

Savin, N. (1978). Pedagogía. La Habana: Pueblo y Educación.

Suárez, J. (1982). El problema de la educación estética en Cuba. La Habana: Editorial Félix Varela.

Ubals, J. (2012). Educación estética y educación artística: Un diálogo no agotado desde la infinitud cercana. Recuperado de https:// blogfcbc.files.wordpress.com/2012/05/educacic3b3n-estc3a9tica-y-educacic3b3n-artc3adstica.pdf

Valle, A. (2012). La investigación pedagógica, otra mirada. La Habana: Pueblo y Educación.

Valdés, M. (1981). Ensayos sobre educación teórica, práctica y experimental. La Habana: Editorial Félix Varela.

Vidal, M. y Fernández, B. (2015). Aprender, desaprender, reaprender. Educación Médica Superior, 29(2). Recuperado de http://www. ems.sld.cu/index.php/ems/article/view/629/260 